\title{
Pemphigus vulgaris presenting as esophageal ulceration. Report of an under- diagnosed manifestation
}

\author{
C. Leal, M. Silva, S. Barbeiro, H. Vasconcelos \\ Department of Gastroenterology, Centro Hospitalar de Leiria, Leiria, Portugal.
}

\begin{abstract}
Pemphigus vulgaris $(\mathrm{PV})$ is a rare autoimmune blistering disorder of the skin and mucous membranes. The true prevalence of esophageal involvement is unknown; esophageal symptoms almost always occur in the context of oral mucosa involvement. We report the case of a 66-year-old man with cutaneous blisters and esophageal symptoms that did not respond to acid suppression therapy. Esophagogastroduodenoscopy showed esophageal ulcers and mucosal desquamation. Biopsies were consistent with the diagnosis of PV. The patient was started on immunosuppressive therapy, achieving remission. This represents a rare case of esophageal involvement of $\mathrm{PV}$ without mucosal involvement and draws attention to a rare cause of dysphagia, which can be fatal if left untreated. (Acta gastroenterol. belg., 2021, 84, 365-366).
\end{abstract}

Key words : endoscopy, pemphigus, esophagus.

\section{Introduction}

Pemphigus is a group of rare blistering disorders characterized by acantholysis, an immune-mediated phenomenon that results in the formation of intraepithelial blisters in mucous membranes and skin. Classically, it is classified as three major forms: pemphigus vulgaris, pemphigus foliaceus and paraneoplastic pemphigus; more recently, IgA pemphigus has also been described. In its most common form, pemphigus vulgaris, the oral cavity is typically involved, being the most common site of mucosal lesions. Esophageal involvement is thought to be rare, especially in the absence of oral lesions. Left untreated, PV can be fatal due to the loss of body fluids and secondary bacterial infections. We report a case of PV with esophageal involvement.

\section{Case report}

A 66-year-old Caucasian man presented to our hospital with blisters on his trunk that had appeared one month ago and a sudden onset of odynophagia and dysphagia the day before. He was already on antihistamine therapy because of the cutaneous manifestations, showing no signs of improvement. His past medical history included arterial hypertension, dyslipidemia and coronary heart disease. Chronic medication included rosuvastatine, clopidogrel, acetylsalicylic acid and bisoprolol. On physical examination, multiple flaccid blisters and erosions on normal-appearing skin were evident, mainly on the trunk, but also on the arms and legs (figure 1). No mucosal involvement was identified at the time. Pruritus was also

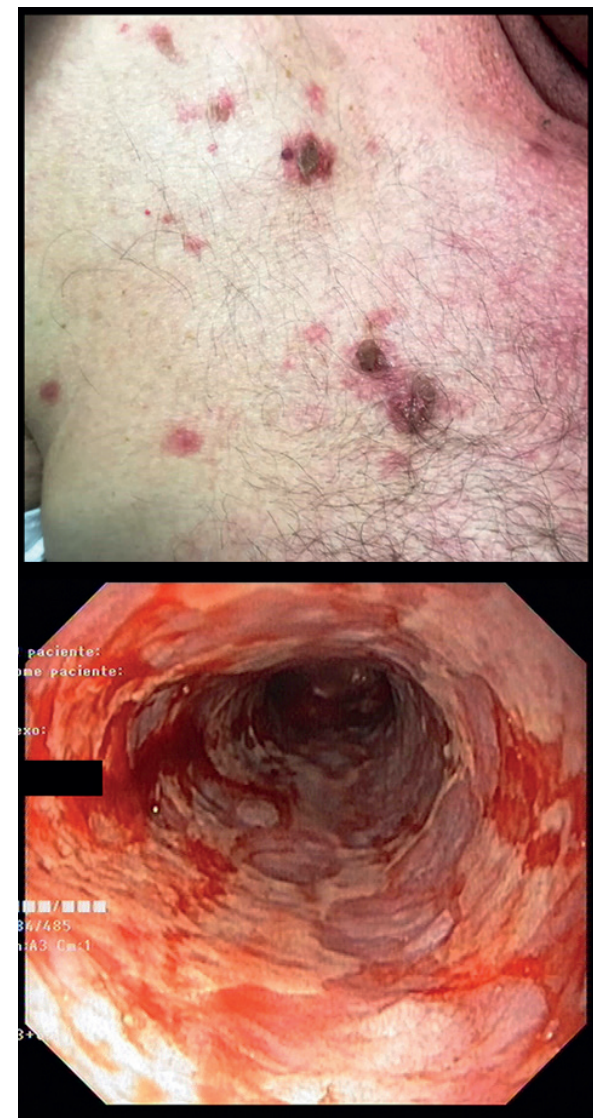

Figure 1. - Pemphigus vulgaris manifestations : blisters and erosions affecting the trunk; and diffuse ulceration in the middle and lower esophagus.

absent. Routine laboratory investigations in-cluding full blood count, renal profile, liver function tests, C-reactive protein and coagulation profile were performed, revealing only a discrete leukocytosis. The patient was referred to an urgent dermatology appointment. A skin biopsy was performed. A hypothesis of bullous pemphigoid was made and the patient was started on systemic corticosteroid and a protein pump inhibitor. Two weeks after the initial evaluation, the skin lesions showed improvement and no mucosal lesions were evident, namely on the oral

Correspondence to : Carina Leal, Department of Gastroenterology, Centro Hospitalar de Leiria, Rua das Olhalvas, 2410-197, Leiria, Portugal.

Email : carina.leal@chleiria.min-saude.pt

Submission date : 14/06/2020

Acceptance date : 06/07/2020 


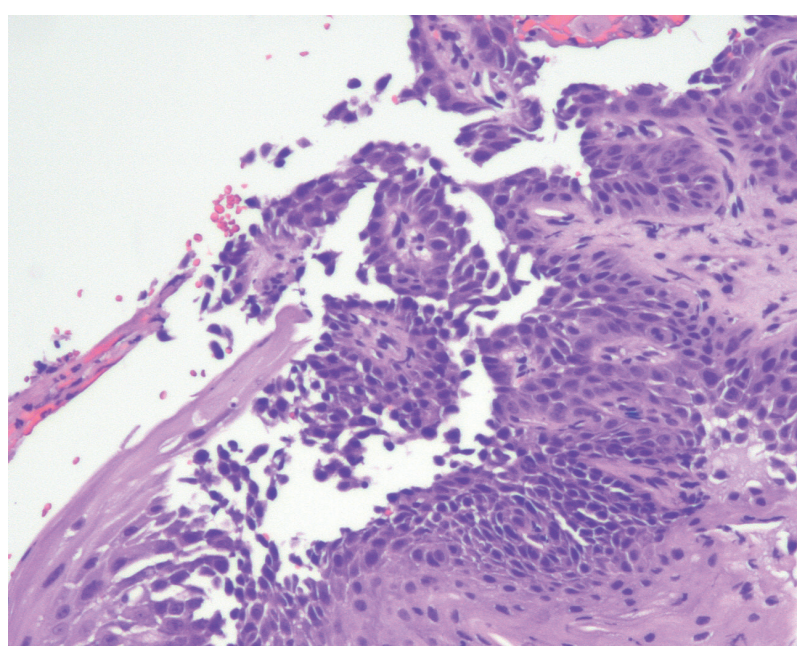

Figure 2. - Suprabasilar acantholysis and intraepithelial clefts seen in magnification (hematoxylin and eosin stain).

cavity. However, the esophageal symptoms worsened, and marked dysphagia and odynophagia were reported. The patient was then referred to our unit for further study. An esophagogastroduodenoscopy was performed. Esophageal mucosa showed marked hyperemia in the upper esophagus and diffuse ulceration in the middle and lower esophagus. Extreme friability was noted (figure A). Biopsies resulted in peeling of linear strips of mucosa. The gastric and duodenal mucosa were preserved. Esophageal biopsies showed signs of ulceration and hemorrhage, suprabasilar acantholysis and intraepithelial clefts (figure 2). Anti-desmoglein autoantibodies 1 and 3 were positive. A diagnosis of PV was made and systemic corticotherapy was started. Azathioprine was further added. The patient showed complete resolution of esophageal symptoms and skin lesions disappeared. Three months after the onset of symptoms an oral erosion appeared. Biopsy of the lesion was consistent with PV. Azathioprine was tapered with complete healing of mucocutaneous erosions.

\section{Discussion}

Pemphigus vulgaris, the most common form of pemphigus, is a rare disease, with incidence rates between 0.1 and 0.5 per 100,000 people per year. The prevalence in men and women is approximately equal and the mean age of onset of disease is 50 to 60 years (1). The hallmark of pemphigus vulgaris is the finding of IgG autoantibodies against glycoproteins desmoglein 1 and desmoglein 3, which play a key role in cell-tocell adhesion. These autoantibodies appear to induce acantholysis, a phenomen that results in easily ruptured blisters and a clinical sign known as the Nikolsky sign. Skin blisters rupture easily, forming painful erosions with little tendency to heal. The oral cavity is the most common site of mucosal involvement, reported in up to $75 \%$ of the cases (2), and often the first site of disease. Lesions in the conjunctivae, nasal mucosa, vagina, labia, penis and anus have also been described. Esophageal involvement, initially thought to be rare, is now thought to be underdiagnosed, as patients may not develop symptoms. Calka et al have performed esophagogastroduodenoscopy in 26 patients with PV, reporting an esophageal involvement in $46.15 \%$ cases (3). The main symptoms, consistent with our report, are dysphagia and odynophagia (4). The most common endoscopic features are blisters, erosions, ulceration and the presence of an easily detached esophageal lining (Nikolsky sign). Differential diagnosis includes other vesiculobullous diseases, paraneoplastic syndromes and drug-induced manifestations. Few cases of esophageal involvement without oral mucosa involvement have been reported in the literature. We found 4 cases in the literature (5-8), oral cavity lesions may not be evident until 2 years of the initial presentation. In this case, oral lesions were present 3 months after the initial diagnosis. Treatment regimens include systemic corticosteroids and immunosuppressive agents. Albeit essential in preventing death from loss of body fluids or secondary bacterial infections, complications from longstanding therapy still occur in $5-10 \%$ of the patients (9).

\section{Conflict of interest}

The authors certify that there is no conflict of interest with any financial organization regarding the material discussed in the manuscript. The authors report no involvement in the research by the sponsor that could have influenced the outcome of this work.

All authors contributed equally to the manuscript and read and approved the final version of the manuscript.

\section{References}

1. KNEISEL A., HERTL, M. Autoimmune bullous skin diseases. Part 1: clinical manifestations. Journal der Deutschen Dermatologischen Gesellschaft 2011, 844-857.

2. ROBINSON JC., LOZADA-NUR F., FRIEDEN I. Oral pemphigus vulgaris: a review of the literature and a report on the management of 12 cases. Oral Surg. Oral Med. Oral Pathol. Oral Radiol. Endod. 1997, 84(4) : 349-55.

3. CALKA O., AKDENIZ N., TUNCER I., METIN A., CESUR RS. Oesophageal involvement during attacks in pemphigus vulgaris patients. Clin. Exp. Dermatol. 2006 Jul, 31(4) : 515-9.

4. GATT K. et al. Superimposed esophageal pemphigus vulgaris and herpes simplex infection. Annals of gastroenterology 2019, 654-654.

5. FAIAS S., LAGE P., SACHSE F. et al. Pemphigus vulgaris with exclusive involvement of the esophagus: case report and review. Gastrointest. Endosc. 2004, $60: 312-15$.

6. WÜRTTENBERGER A, BARNERT J, WIENBECK M. Long-lasting odynophagia and haematemesis - the only clinical signs of a pemphigus vulgaris. $Z$. Gastroenterol. 2002, 40 : 419-24.

7. SALEM Z., STRIPPOLI A., DEPASQUALE JR. Isolated esophageal pemphigus vulgaris. Am J Gastroenterol 2003;98:201.

8. AL-JANABI A, GREENFIELD S. Pemphigus vulgaris : a rare cause of dysphagia. Case Reports 2015, 2015 : bcr2015212661.

9. AHMED AR, MOY R. Death in pemphigus. J. Am. Acad. Dermatol. 1982, 7 : 221-8. 